\title{
Gradiente y ancho del timpanograma en niños de 4 a 6 años
}

\author{
IVÁN NOVOA C. ${ }^{1}$, DANISA TORRES M. ${ }^{2}$ \\ 1. Tecnólogo Médico del Hospital Militar de Santiago General Brieba Arán. \\ 2. Tesista de la Carrera de Tecnología Médica, Mención Otorrinolaringología, Universidad de Chile 2003.
}

\begin{abstract}
Tympanogram gradient and width in Children 4 to 6 years-old

Background: Impedanciometry is a sensitive test that allows the diagnosis of intratimpanic effusion. Both tympanogram gradient (TG) and tympanogram width (TW) can be obtained from it. Usually, international reference values for these variables are used, but their distribution in chilean pediatric population remains uncertain. Objective: Establish normal values for these parameters in chilean children 4 to 6 years-old. Method: This study was performed at the Otorhinolaryngology Service of Hospital Militar in Santiago, contemplating all chilean army personnel children, both sexes, 4 to 6 years-old who assisted to the center between March and June 2003. 180 children were evaluated by anamnesis, otoscopy, audiometry and impedanciometry. Results: Normal values for TG were determined with a $95 \%$ confidence interval. These were 0,21 to 0,62 for girls and 0,22 to 0,57 for boys. Percentiles were determined for TW, where its normal value can be estimated in our population by means of its inverse, with significative correlation with TG. Conclusion: These normal values will improve impedanciometry accuracy in the diagnosis of intratimpanic effusion, at the moment based on timpanometric shape and compliance peak.

(Key words: tympanogram, width, gradient, impedanciometry).

Rev Chil Pediatr 2009; 80 (2): 137-143
\end{abstract}

\section{RESUMEN}

Introducción: La impedanciometría es un estudio sensible para la pesquisa precoz de efusión intratimpánica que permite obtener las variables gradiente (GT) y ancho del timpanograma (AT). Generalmente, se utilizan valores de referencia internacionales de dichas variables para este propósito, pues se desconoce su distribución en la población pediátrica chilena. Objetivo: Establecer valores de normalidad de estos parámetros en niños chilenos entre 4 y 6 años. Pacientes y Método: Este estudio se realizó en el Servicio de Otorrinolaringología del Hospital Militar de Santiago. Población: todos los niños y niñas entre 4 y 6 años, hijos del personal del Ejército de Chile, asistentes al hospital entre marzo y junio de 2003. Muestra: 180

Trabajo recibido el 29 de abril de 2008, devuelto para corregir el 28 de octubre de 2008, segunda versión el 29 de enero de 2009, aceptado para publicación el 16 de febrero de 2009 .

Ejecución de la Investigación: Servicio de Otorrinolaringología y Laboratorio de Otoneurología del Hospital Militar de Santiago General Brieba Arán.

Equipos: Audiómetro: Interacoustics AC 40 ${ }^{\circledR}$, calibración ANSI 69. Impedanciómetro: Madsen Electronics ${ }^{\circledR}$, modelo Zodiac 901. Ambos del Hospital Militar de -Santiago.

E-mail: danisa.torres@hotmail.com 
niños, evaluados mediante anamnesis, otoscopia, audiometría e impedanciometría. Resultados: Valores normales de GT: 0,21 a 0,62 en niñas y 0,22 a 0,57 en niños. Ambos con un intervalo de confianza de 95\%. Para AT se determinaron percentiles y su valor de normalidad se estima mediante la correlación inversa existente entre GT y AT, que es significativa. Conclusión: Estos valores de normalidad aumentarán la precisión impedanciométrica en el diagnóstico de efusión intratimpánica, que actualmente, en Chile, se basa en la forma timpanométrica y peak de complianza.

(Palabras clave: timpanograma, ancho, gradiente, impedanciometría).

Rev Chil Pediatr 2009; 80 (2): 137-143

\section{Introducción}

La efusión intratimpánica es la acumulación de líquido dentro del oído medio. Esta alteración es común, especialmente en niños, y es causada por múltiples factores. Puede manifestarse con síntomas como otalgia, plenitud ótica, autofonía y acúfenos, y provocar una disminución de la audición. Además, la efusión intratimpánica puede traer complicaciones como atrofia de tímpano, erosión de los huesecillos, tímpanoesclerosis, otitis media crónica o granulomas de colesterol, llegando a afectar incluso el oído interno ${ }^{1}$. Por estas razones es necesario utilizar todas las herramientas diagnósticas para su detección y tratamiento precoz, sobre todo en niños, quienes son propensos a presentar esta patología, que si no es detectada y tratada a tiempo puede provocar a largo plazo una disminución en la audición que, entre otras complicaciones, interfiere con el desarrollo del lenguaje, proceso que en el rango de edad en estudio se encuentra en plena evolución.

El diagnóstico presuntivo de la efusión intratimpánica se apoya en la historia clínica, otoscopia, otomicroscopia, audiometría tonal e impedanciometría. Pero estas herramientas son insuficientes por sí solas para diagnosticar esta patología y sólo en la medida en que se combinan adecuadamente, se obtiene un menor número de falsos positivos y falsos negativos. Sin embargo, hay consenso en que la impedanciometría sería el medio más precoz, sensible y exacto para pesquisar la efusión intratimpánica ${ }^{2-4}$.

La timpanometría es una técnica usada para evaluar la movilidad y la condición de la membrana timpánica y del oído medio durante las variaciones de presión en el conducto auditivo externo ${ }^{5}$. Así, Jerger (1970), denominó curva tipo "A" al timpanograma que presenta su punto de máxima complianza centrado alrededor de la presión 0 entre $\pm 100 \mathrm{daPa}$, lo que indica que hay ausencia de líquido en oído medio, y curva tipo "B" a aquella curva plana, que no alcanza un punto de máxima complianza, lo que indica la presencia de líquido dentro en medio (figura 1). Sin embargo, en la práctica se han detectado pacientes que aún teniendo curvas tipo "A", éstas son lo suficientemente anchas y que sin ser planas nos hacen sospechar una efusión intratimpánica, lo que se ha comprobado a través de paracentesis ${ }^{2}$.

Del timpanograma se obtiene la altura relativa de la curva, la complianza estática y la presión del punto de máxima complianza ${ }^{5-8}$, parámetros comúnmente usados para este propósito en nuestro país. En Inglaterra y Estados Unidos se suman a esta batería de evaluación timpanométrica los valores de gradiente y ancho del timpanograma, parámetros que son un

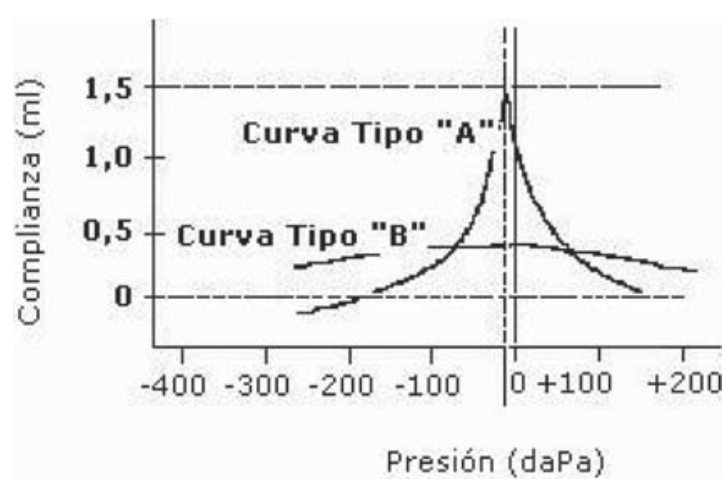

Figura 1. Curvas tipo "A" y "B" según Jerger (1970). 

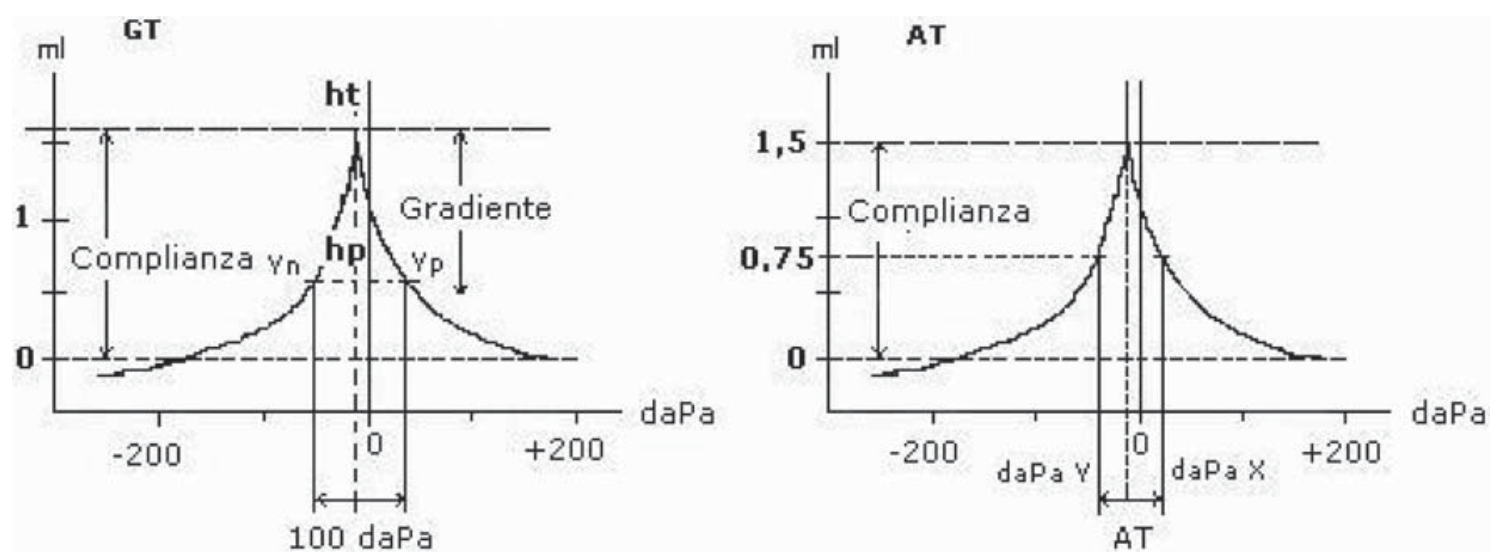

Figura 2. Gradiente (GT) y Ancho del Timpanograma (AT).

resumen de la forma del timpanograma y por lo tanto, permiten entregar un diagnóstico más exacto de efusión intratimpánica en comparación con otras mediciones timpanométricas ${ }^{9}$ y si bien están normados en dichos países, no han sido estandarizados en nuestra población infantil.

\section{Gradiente y Ancho del Timpanograma:} La gradiente se expresa como: GT $=\mathbf{h p} / \mathbf{h t}$ y corresponde la relación entre la distancia vertical medida desde el punto de máxima complianza hasta una línea horizontal que intercepta el timpanograma en dos puntos que están separados entre sí en $100 \mathrm{daPa}(\mathrm{hp})$, y la altura total de la misma curva (ht $)^{2}$. El ancho del timpanograma se expresa así: $\mathbf{A T}=\mathbf{d a P a} \mathbf{X}-\mathbf{d a P a}$ $\mathbf{Y}, \mathrm{y}$ corresponde a la diferencia de presión entre dos puntos ( $\mathrm{X}$ e $\mathrm{Y}$ ) de la curva timpanométrica en la cual la complianza corresponde a la mitad del punto de máxima complianza (Guidelines for Screening for Hearing Impairment and Middle Ear Disorders., ASHA 1990) (figura 2).

El objetivo de este trabajo es determinar valores de normalidad para GT y AT en la población infantil de 4 a 6 años de edad, según sexo.

\section{Pacientes y Métodos}

Estudio descriptivo realizado en el Departamento de Otoneurología del Servicio de Otorri- nolaringología del Hospital Militar de Santiago. La población en estudio estuvo constituida por todos los niños de 4 a 6 años de edad, de ambos sexos, hijos del personal del Ejército de Chile, que asistieron al Hospital Militar de Santiago, en el período de marzo a junio de 2003. De ellos, se seleccionó una muestra de 180 niños de ambos sexos, 90 niñas y 90 niños, quienes cumplieron con los criterios de inclusión, los cuales participaron en el estudio previa autorización de los padres, quienes leyeron y firmaron un documento de consentimiento informado (anexo A), aprobado por el comité de ética del hospital.

\section{Criterios de inclusión}

* Se realizó una anamnesis completa a niños y padres para descartar alguna patología de oído medio. Ésta contempló preguntas acerca de la presencia de signos y síntomas de afección auditiva (presencia de otitis, supuración o dolor de oídos) y sus antecedentes mórbidos, pérdida auditiva, síndrome que conlleve mal desarrollo de la trompa de Eustaquio y episodios recurrentes de otitis, y presencia de fisura palatina actual o tratada.

- Presentar una otoscopia normal, esto es, conducto auditivo externo y membrana timpánica indemne (sin congestión, perforación, retracción y/o tumoraciones asociadas). Los niños que presentaron tapón de cerumen fueron derivados al médico otorrino para que éste fuese extraído. 
- Presentar barrido frecuencial audiométrico positivo a $20 \mathrm{~dB}$ entre 250 y $4000 \mathrm{~Hz}$. Éste se realizó en una cámara sonoamortiguada con un audiómetro clínico Interacoustics AC$40^{\circledR}$ calibrado según las normas vigentes (ANSI 69).

- Cada niño debió presentar curvas tipo "A", con reflejos positivos ipsi y contralaterales inferiores a $100 \mathrm{~dB}$. Esta evaluación se realizó con el impedanciómetro Madsen Electronics, modelo Zodiac $901^{\circledR}$.

- En cada etapa de la evaluación se les explicó al niño y a sus padres en qué consistía el procedimiento y las medidas a tomar en caso de alguna alteración evidenciada en el examen, de ocurrir esto último, se aconsejó a los padres llevar a su hijo al otorrinolaringólogo.

Para evitar sesgos de procedimiento se calibraron periódicamente los equipos según las normas vigentes, se dieron las mismas instrucciones a cada niño antes de iniciar la toma de exámenes, todas las mediciones necesarias para cada paciente fueron realizadas siempre por el mismo examinador. Las mediciones se realizaron siempre en los mismos equipos.

La unidad de observación fue cada niño estudiado. Se consideró como unidad de análisis, cada oído; se evaluó una población de 424 oídos, de ellos, se excluyeron 82; 31 de niñas $(37,8 \%)$ y 51 de niños $(62,2 \%)$. Las causas de exclusión se detallan en la tabla 1. Los oídos que formaron parte de la muestra fueron 342 .

Los datos fueron procesados computacionalmente en planillas Excel ${ }^{\circledR}$, obteniéndose las medidas de resumen correspondientes (pro- medio, desviación estándar y percentiles). Las variables a analizar fueron GT y AT y su distribución por sexo y oído; se determinó la normalidad de las curvas aplicando la prueba de Shapiro-Wilk. Para determinar la influencia del sexo y diferencia por oídos en GT se utilizó la prueba t-student para dos muestras independientes. Para determinar la influencia del sexo y la diferencia por oídos en AT se empleó la prueba de Wilcoxon. Para establecer un modelo de valor predictivo, se realizó un estudio de correlación de Spearman y regresión lineal con análisis de varianza. Todas las pruebas con un error $\mathrm{p}<0,05$.

\section{Resultados}

Distribuciones de frecuencias de GT según sexo: La GT se registró en 342 oídos: 50\% oídos derechos y $50 \%$ oídos izquierdos. El comportamiento de GT entre ambos sexos es significativamente diferente según la prueba de t-student para dos muestras independientes. Según la prueba de Shapiro-Wilk, las curvas para ambos sexos se distribuyen normal, con un $\mathrm{W}=0,98831$ y un $p$ de $\mathrm{W}=0,16886$ en niñas $\mathrm{y}$ con un $\mathrm{W}=0,98964$ y un $p$ de $\mathrm{W}=0,24719$ en niños (tabla 2). Se establecen límites de normalidad en base a la media y a la desviación estándar para GT según sexo con un nivel de confianza del 95\%. Estos límites son: 0,21-0,62 en niñas y $0,22-0,57$ en niños.

Distribuciones de frecuencias de AT según sexo: El AT se registró en 342 oídos: 50\% oídos derechos y $50 \%$ oídos izquierdos. Se estableció una diferencia significativa del com-

Tabla 1. Causas de exclusión de la muestra

\begin{tabular}{|c|c|c|c|c|c|c|}
\hline \multirow[t]{2}{*}{ Causa de exclusión } & \multicolumn{2}{|c|}{ Niñas } & \multicolumn{2}{|c|}{ Niños } & \multicolumn{2}{|c|}{ Total } \\
\hline & $\mathbf{n}$ & $\%$ & $\mathbf{n}$ & $\%$ & $\mathbf{n}$ & $\%$ \\
\hline Disfunción tubaria & 14 & 45,1 & 24 & 47,1 & 38 & 46,3 \\
\hline Reflejos negativos & 6 & 19,3 & 10 & 19,6 & 16 & 19,5 \\
\hline Otitis en los últimos 3 meses & 3 & 9,7 & 8 & 15,7 & 11 & 13,4 \\
\hline Tímpano congestivo & 3 & 9,7 & 5 & 9,8 & 8 & 9,8 \\
\hline Hipoacusia & 2 & 6,5 & 2 & 3,9 & 4 & 4,9 \\
\hline Reflejos $\mathrm{a} \geq 100 \mathrm{~dB}$ & 3 & 9,7 & 0 & 0 & 3 & 3,7 \\
\hline Curva Ad & 0 & 0 & 2 & 3,9 & 2 & 2,4 \\
\hline Total & 31 & 100 & 51 & 100 & 82 & 100 \\
\hline
\end{tabular}


Tabla 2. Distribución de GT según sexo

\begin{tabular}{|c|c|c|c|c|c|c|}
\hline \multirow{2}{*}{ Gradiente del timpanograma } & \multicolumn{2}{|c|}{ Niñas } & \multicolumn{2}{|c|}{ Niños } & \multicolumn{2}{|c|}{ Total } \\
\hline & $\mathbf{n}$ & $\%$ & $\mathbf{n}$ & $\%$ & $\mathbf{n}$ & $\%$ \\
\hline $0,17-0,22$ & 4 & 2,3 & 7 & 4,1 & 11 & 3,2 \\
\hline $0,23-0,28$ & 8 & 4,7 & 17 & 9,9 & 25 & 7,3 \\
\hline $0,29-0,34$ & 35 & 20,5 & 35 & 20,5 & 70 & 20,5 \\
\hline $0,35-0,40$ & 36 & 21,1 & 38 & 22,2 & 74 & 21,6 \\
\hline $0,41-0,46$ & 30 & 17,5 & 39 & 22,8 & 69 & 20,2 \\
\hline $0,47-0,52$ & 30 & 17,5 & 20 & 11,7 & 50 & 14,6 \\
\hline $0,53-0,58$ & 20 & 11,7 & 13 & 7,6 & 33 & 9,6 \\
\hline$\geq 0,59$ & 0 & 4,7 & 2 & 1,2 & 10 & 3,0 \\
\hline Total & 171 & 100,0 & 171 & 100,0 & 342 & 100,0 \\
\hline Promedio & 0,42 & & 0,39 & & 0,40 & \\
\hline Desviación estándar & 0,10 & & 0,10 & & 0,10 & \\
\hline
\end{tabular}

Tabla 3. Distribución de AT según sexo

\begin{tabular}{|c|c|c|c|c|c|c|}
\hline \multirow{2}{*}{ Ancho del timpanograma } & \multicolumn{2}{|c|}{ Niñas } & \multicolumn{2}{|c|}{ Niños } & \multicolumn{2}{|c|}{ Total } \\
\hline & $\mathbf{n}$ & $\%$ & $\mathbf{n}$ & $\%$ & $\mathbf{n}$ & $\%$ \\
\hline $58-78$ & 4 & 2,3 & 2 & 1,2 & 6 & 1,8 \\
\hline $79-99$ & 31 & 18,1 & 20 & 11,7 & 51 & 14,9 \\
\hline $100-120$ & 53 & 31,0 & 39 & 22,8 & 92 & 26,9 \\
\hline $121-141$ & 38 & 22,2 & 53 & 31,0 & 91 & 26,6 \\
\hline $142-162$ & 32 & 18,7 & 24 & 14,0 & 56 & 16,4 \\
\hline $163-183$ & 7 & 4,1 & 23 & 13,5 & 30 & 8,8 \\
\hline$\geq 184$ & 6 & 3,6 & 10 & 5,8 & 16 & 4,6 \\
\hline Total & 171 & 100,0 & 171 & 100,0 & 342 & 100,0 \\
\hline Promedio & 124,3 & & 133,7 & & 129,0 & \\
\hline Desviación estándar & 30,3 & & 29,6 & & 30,6 & \\
\hline percentil 2,5 & 79,3 & & 83,5 & & 81,0 & \\
\hline percentil 25 & 103,0 & & 112,0 & & 107,3 & \\
\hline percentil 50 & 120,0 & & 130,0 & & 126,0 & \\
\hline percentil 75 & 142,5 & & 150,5 & & 147,0 & \\
\hline percentil 97,5 & 194,3 & & 201,8 & & 201,5 & \\
\hline
\end{tabular}

portamiento de AT entre ambos sexos (Wilcoxon, $\mathrm{p}<0,05)$. Se aplicó prueba de Shapiro-Wilk, que verificó que el AT para los grupos de niñas y niños no se distribuye normal ( $\mathrm{p}$ de $\mathrm{W}<0,05$. Tabla 3 ). La distribución de AT se establece a través de los percentiles 2,$5 ; 25$, 50,75 y 97,5 . De acuerdo con esto, el $95 \%$ del AT en el grupo de niñas está entre 79,3 y 194,3 daPa, y en el grupo de niños, entre 83,5 y 201,8 daPa.

Distribuciones de frecuencias de GT y AT según oído: El comportamiento de GT y AT según oído es estadísticamente diferente y significativo, Wilcoxon, $\mathrm{p}<0,05$.
Correlación entre Gradiente y Ancho del Timpanograma: Se determina la correlación inversa entre GT y AT, mediante el coeficiente de correlación de Spearman, que en niñas es 0,987 y en niños, $-0,97$, ambos con un intervalo de confianza de un $95 \%$. El estudio de regresión lineal para establecer un modelo de predicción del comportamiento de estas variables en cada sexo, establece que la ecuación de la recta para niñas es: $\hat{Y}=0,8216-0,0033 x$ y para niños es $\hat{Y}=0,7867-0,003 x$. El análisis de varianza establece que el modelo es un buen predictor, explicando el $97,4 \%$ de la variabilidad en niñas y el $94,1 \%$ de la variabilidad en niños. 


\section{Discusión}

Como se mencionó anteriormente, la impedanciometría es un método objetivo aceptado para el diagnóstico de efusión intratimpánica. En este punto coinciden muchos especialistas, quienes confirman que este examen es tremendamente útil junto con un examen físico para realizar tamizado en una gran población de niños y presenta validez como herramienta diagnóstica en efusión intratimpánica ${ }^{3}$. Esto tiene vital importancia, pues permite contar con cifras objetivas para establecer planes de intervención en salud.

La altura relativa de la curva, la complianza estática y la presión del punto de máxima complianza son parámetros comúnmente usados para este propósito. Además, se ha demostrado que GT y AT, y la presión en la cual se alcanza la máxima complianza son reproducibles en múltiples mediciones ${ }^{10}$. Sin embargo, es difícil llevar a cabo una correcta comparación de varios parámetros timpanométricos para el diagnóstico preciso de la efusión intratimpánica, ya que no existe un valor de referencia timpanométrico definido para esta patología cuando el timpanograma arroja una curva tipo "A". En este contexto, el diagnóstico de la efusión por otoscopia o paracentesis es usado como un estándar para la validez de los resultados de la timpanometría ${ }^{11-13}$.

El objetivo de este estudio fue establecer valores de normalidad de GT y AT en niños de 4 a 6 años, según sexo. Sin embargo, encontramos que la distribución de AT en niñas y niños no es normal, pero GT sí se distribuye de dicha forma en ambos sexos, lo que permite establecer límites de normalidad de esta variable en sujetos entre 4 y 6 años, valores que no habían sido documentados para este grupo de edad en nuestro país.

Brooks en 1968, estableció como límite inferior de GT en niños un valor de 0,03 para distinguir entre presencia o ausencia de efusión intratimpánica ${ }^{14}$. Nosotros establecimos límites de normalidad que se encuentran entre $0,21 \mathrm{y}$ 0,62 en niñas y, entre 0,22 y 0,57 en niños, valores diferentes a los encontrados por Brooks. Estas diferencias ya han sido documentadas por Koebsell y Margolis en 1986, y la atribuyen a las unidades entregadas por el equipo con el cual Brooks realizó sus estudios, que aparentemente entregaba valores en unidades de complianza relativa ${ }^{15}$.

La estandarización de AT en niños de 3 a 5 años documentada por la ASHA en 1990 establece como límite superior de normalidad 100 $\mathrm{daPa}$, un valor superior a éste es indicador de efusión intratimpánica ${ }^{16}$. En nuestro estudio, como AT no se distribuyó normal, establecimos percentiles para conocer el comportamiento de su distribución.

En la práctica, se ha observado que a valores aumentados de AT le corresponden valores disminuidos de GT. Esto nos permitió establecer la correlación inversa entre GT y AT según sexo la cual es significativa y el análisis de varianza de la regresión lineal para dichos grupos establece que el modelo es un buen predictor. El alto valor predictivo de esta herramienta es importante en clínica pues permite establecer un valor de GT dado un determinado valor de AT a través de una ecuación de la recta, con esto podemos extrapolar los valores de normalidad de AT para cada sexo según los valores de normalidad de GT respectivos; esta relación no había sido documentada en niños chilenos.

Se encontró una diferencia significativa de GT para ambos sexos, este mismo hallazgo se verificó en AT $(p<0,05)$. Investigaciones anteriores (Wiley y cols, 1966; Blood y cols, 1977 y Roup y cols, 1998) informaron acerca de este hallazgo para GT en adultos, pero su causa no fue explicada. La diferencia entre sexos de ambas variables se podría atribuir a diferencias anatómicas y a sus cambios durante el crecimiento. Este último punto ha sido estudiado por Margolis y Heller en 1987, quienes encontraron diferencias significativas en el valor de GT en diferentes grupos etéreos. Este mismo fenómeno podría explicar la diferencia entre oídos para ambas variables cuando hablamos de una población pediátrica, no así en adultos, en que no se observan diferencias significativas para GT según oído en una población universitaria chilena en un rango de edad entre los 18 a 29 años ${ }^{17}$.

Este trabajo pretende ser un punto de referencia para futuras investigaciones en la aplicación clínica de estos parámetros, especialmente en niños, grupo que va sufriendo cambios ana- 
tómicos del oído medio con el crecimiento, y en los cuales ya se han documentado diferencias según sexo y según oído en estas variables, en grupos de edades específicos.

\section{Conclusiones}

- Se establecen límites de normalidad para GT según sexo, los cuales varían entre 0,21 y 0,62 en niñas y, entre 0,22 y 0,57 en niños.

- La distribución de AT en niñas y niños se establece a través de los percentiles 2,$5 ; 25$, 50,75 y 97,5 . De acuerdo con esto, el $95 \%$ del AT en el grupo de niñas está entre 79,3 y $194,3 \mathrm{daPa}$, y en el grupo de niños, entre 83,5 y $201,8 \mathrm{daPa}$.

- Se determina que las variables en estudio según sexo y oído, son estadísticamente diferentes $(\mathrm{p}<0,05)$.

- Existe correlación inversa y significativa entre el AT y GT según sexo, siendo el coeficiente de correlación de Spearman en niñas: $-0,987$, y en niños: $-0,97$.

- Se establece mediante el modelo de regresión lineal, que la correlación entre estas variables tiene un alto valor predictivo para la GT dado un valor de AT, explicando el 97,4 y $94,1 \%$ de la variabilidad en niñas y niños, respectivamente. Mediante este modelo, además se pudo establecer las siguientes ecuaciones de la recta para GT dado un valor de AT según sexo:

- Niñas: $G T=0,8216-0,0033^{*} A T$

- Niños: $G T=0,7867-0,0030 * A T$

El conocimiento de estos datos aumentará la precisión impedanciométrica para el diagnóstico de efusión intratimpánica, que actualmente, en Chile, se basa en la forma timpanométrica y peak de complianza.

\section{Referencias}

1.- Paparella M, Jung T, Goycolea V: Otitis media con derrame. En: Otorrinolaringología: Otología y Neurootología, $3^{\circ}$ Edición, Vol. 2, Buenos Aires: Editorial
Médica Panamericana, 1994; 1543-73.

2.- Emhart C, García M, May A: Variaciones audiométricas e impedanciométricas en efusiones intratimpánicas. Rev Otorrinolaring 1979; 39: 56-64.

3.- Bluestone D, Berry C, Paradise L: Audiometry and timpanometry in relation to middle ear effusions in children. Laryngoscope 1973; 83: 594-604.

4.- Katz J: Handbook of Clinical Adiology. $5^{\circ}$ Edición, Baltimore Maryland: Editorial Williams \& Wilkins, 2002; 183-5.

5.- Lidén G, Peterson J, Björkman G: Tympanometry: A method for analysis of middle-ear function. En: Selected Readings in Impedance Audiometry, University of Colorado Medical Center, New York, 1976; 12632.

6.- Michael B, Ferry $W$ : Overview and basic principles of acoustic immittance. En: Handbook of Clinical Adiology, $4^{\circ}$ Edición, Baltimore Maryland: Editorial Williams \& Wilkins, 1994; 271-82.

7.- Hall W, Chandler D: Tympanometry in clinical audiology. En: Handbook of Clinical Adiology, $4^{\circ}$ Edición, Baltimore Maryland: Editorial Williams \& Wilkins, 1994; 283-99.

8.- Northern L, Rock H, Frye D: A technique for identifying ear disease in children. En: Selected Readings in Impedance Audiometry, University of Colorado Medical Center, New York, 1976; 140-7.

9.- De Jonge $R$ : Normal tympanometric gradient: a comparasion the three methods. Audiology 1986; 25: 299-308.

10.- Gaihede M, Ovesen T: Precision of tympanometric measurement. J Speech Lang Hear Res 1997; 40: 215 22.

11.- Paradise L, Smith G, Bluestone D: Tympanometric detection of middle ear effusion in infants and young children. Pediatrics 1976; 58: 198-210.

12.- Nozza J, Bluestone D, Kardatzke D, Bachman R: Identification of middle ear effusion by aural acoustic admittance and otoscopy. Ear Hear 1994; 15 (4): 31023.

13.- Margolis H, Hunter L, Giebink S: Tympanometric evaluation of middle ear function in children with otitis media. Otol Rhinol Laryngol 1994; 103: 34-8.

14.- Brooks D: An objective method of detecting fluid in the middle ear. Audiology 1968; 7: 280-6.

15.- Koebsell A, Margolis H: Tympanometric gradient measured from normal preschool children. Audiology 1986; 25: 149-57.

16.- Hall W, Chandler D: Tympanometry in clinical audiology. En: Handbook of Clinical Adiology, $4^{\circ}$ Edición, Baltimore Maryland: Editorial Williams \& Wilkins, 1994; 283-99.

17.- Allende M, Jofré C, Pavez E: Valores de normalidad de la gradiente de Brooks en la población estudiantil universitaria. Tesis para optar al título de tecnólogo médico. Universidad de Chile. 2001. 